\title{
DETERMINACIÓN DE CUBIERTAS INCLINADAS PARA PROGRAMAS DE ARQUITECTURA
}

\author{
(DETERMINATION OF SLOPED ROOFS FOR ARCHITECTURAL PROGRAMS)
}

Alfonso Recuero y José Pedro Gutiérrez, Drs. Ings. de C.C. y P.

ICCET/CSIC

Fecha de recepción: $15-\mathrm{X}-92$

ESPAÑA

403-14

\section{RESUMEN}

Se presenta un algoritmo que permite determinar una cubierta inclinada sobre una planta poligonal plana de forma cualquiera simple o múltiplemente conexa, esto es, considerando patios interiores. La inclinación de cada faldón se fija independientemente. lo cual permite resolver tanto los casos de cubiertas igualmente inclinadas, como el de cubiertas de cumbreras de altura constante, el de cubiertas amansardadaso el de fachadas sin faldones. El algoritmo proporciona la descripción geométrica de cada uno de los faldones independientemente, lo que facilita su incorporación en programas para diseño de edificios asistido por computador.

En el presente trabajo se incluye una descripción exhaustiva del algoritmo, así como un conjunto representativo de ejemplos de utilización.

\section{SUMMARY}

The article presents an algorithm which allows the determination of a sloped roof over a plane polygonal plan of any simply or multiply connected form, that is to say, considering the interior courtyards. The slope of each gable is fixed independently, which allow's us to solve a variety of cases, such as those of equally sloped roofs. roofs with constant height ridges, attic roofs or roofs over façades without gables. The algorbitm provides the geometric description, of each gable separately, which facilitates its incorporation in the computer building design programs.

This work includes a thorough description of the algorithm as well as a representative set of practical examples.

\section{Introducción}

Los actuales programas para el diseño de edificios asistido por computador suelen adolecer de la falta de un procedimiento general para la determinación automática de las cubiertas inclinadas, resolviendo exclusivamente los casos más simples, y debiendo ser el proyectista quien, en casos más complejos, resuelva manualmente las intersecciones entre los faldones de la cubierta.

En el presente trabajo se describe un procedimiento automático que permite resolver con gran generalidad la cubierta inclinada sobre una base poligonal plana. Permite considerar contornos exteriores del edificio cualesquiera, así como la existencia de uno o varios patios interiores que pueden estar a cotas distintas. Al describir independientemente la inclinación de cada uno de los faldones, permite resolver todos los casos que se presenten en la práctica, por ejemplo, considerar fachadas sin faldón (cubierta a dos aguas), cubiertas igualmente inclinadas, cubiertas con cumbreras a una altura prefijada en plantas de borđes opuestos paralelos (que puede obligar a que los faldones tengan inclinaciones diferentes), o cubiertas con 
faldones con dos inclinaciones distintas (cubiertas amansardadas). El resultado producido por el algoritmo es la descripción independiente de cada uno de los faldones de la cubierta, mediante la secuencia ordenada de las coordenadas de sus vértices, que puede ser fácilmente interpretada por cualquier programa de diseño de edificios asistido por computador.

Las perspectivas presentadas en este trabajo han sido obtenidas incorporando este algoritmo al programa CADET, sistema no comercial desarrollado por el Instituto $(1,2)$.

Este trabajo forma parte del proyecto "Desarrollo de sistemas CAD para el proyecto completo de estructuras de hormigón" financiado por la DGICYT (PB 0344), y cuya realización se lleva a cabo en el Instituto de Ciencias de la Construcción Eduardo Torroja.

\section{Objeto del algoritmo}

El algoritmo que se presenta tiene por objeto determinar la descripción de una cubierta inclinada sobre un edificio de planta poligonal cualquiera que permita cubrir la mayoría de los casos prácticos. Requiere, exclusivamente como datos, la descripción de la base mediante la definición de sus contornos, y la inclinación de cada uno de sus faldones. Proporciona como resultados la descripción geométrica independiente de cada uno de los faldones de la cubierta, mediante la secuencia de los vértices que definen cada uno de ellos.

Los resultados se dan de la forma más compacta posible, esto es, utilizando el menor número posible de vértices numerados de forma correlativa con objeto de facilitar su incorporación en forma de subrutina a cualquier programa de diseño asistido por computador de edificios.

Para su implementación, el algoritmo ha sido desarrollado en RM Basic e incorporado como subrutina externa al sistema no comercial CADET, desarrollado en el ICCET.

\section{Descripción de los datos}

Los datos de partida del problema son la descripción de la planta y la inclinación de cada faldón, descripción que debe ser compacta y completa. La planta de la cubierta se supone definida por un conjunto de polígonos planos horizontales, de los que la parte a cubrir puede ser el interior o el exterior, pudiendo además cada polígono estar situado a una cota distinta. Los faldones de la cubierta se suponen definidos por un plano que pasa por una
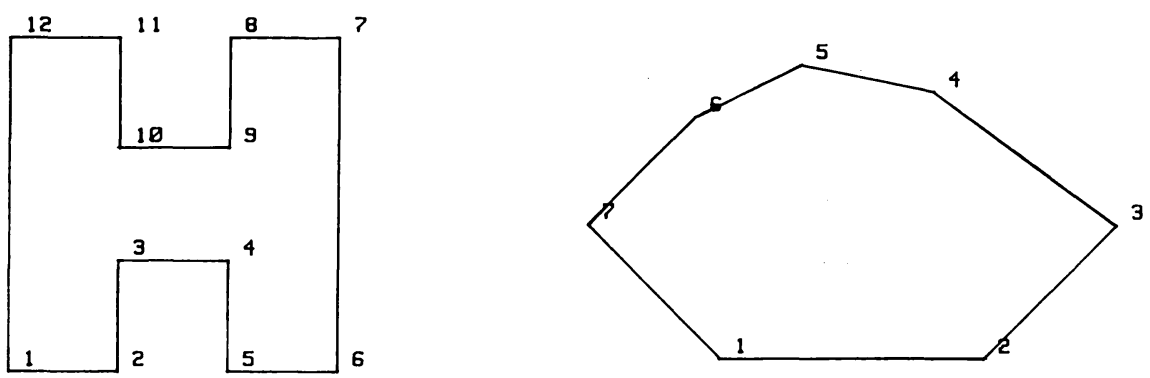

SIMPLEMENTE CONEXA
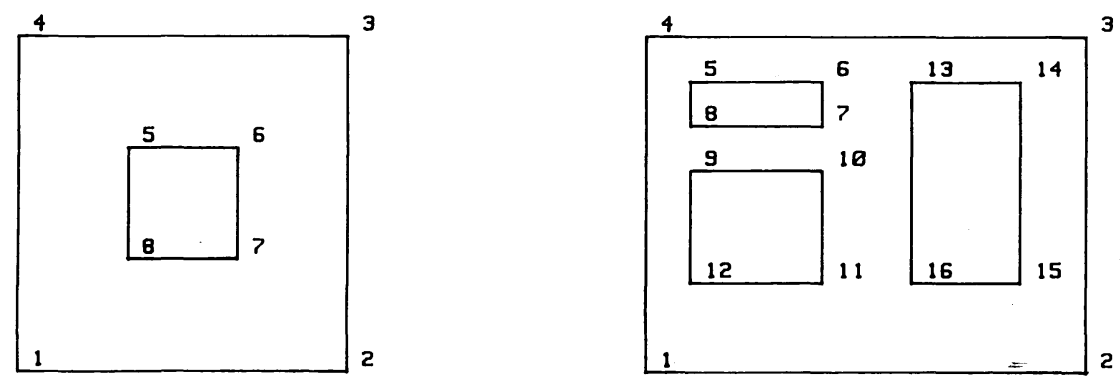

MULTIPLEMENTE CONEXA 
arista de uno de los polígonos que definen la base, que tiene una cierta inclinación subiendo o bajando respecto de la cota de la arista de base. Puede ocurrir que haya aristas de base sobre las que no hay faldones, en las que la fachada se prolonga verticalmente. El formato de datos que se describe a continuación permite realizar esta descripción completa y compacta:

- Número de bordes de la planta. En el caso de ser simplemente conexa habrá un solo borde; en el caso de existir patios interiores debería utilizarse un borde para describir el contorno de cada uno de los patios.

- Para cada uno de los bordes de la planta deberá darse el número de vértices que lo definen y la cota del borde, y para cada uno de estos vértices sus coordenadas $\mathrm{X}$ e $\mathrm{Y}$, así como la inclinación que forma el faldón correspondiente con el plano horizontal medido en grados sexagesimales. Al recorrer un lado el faldón se considera situado a la izquierda, de modo que si el borde representa un contorno exterior el sentido de recorrido debe ser antihorario, en tanto que si representa un patio el sentido del recorrido debe ser horario. Si el ángulo del faldón es positivo, el faldón se eleva sobre el borde, en tanto que si es negativo el faldón desciende desde el borde. Cada faldón se asocia con el

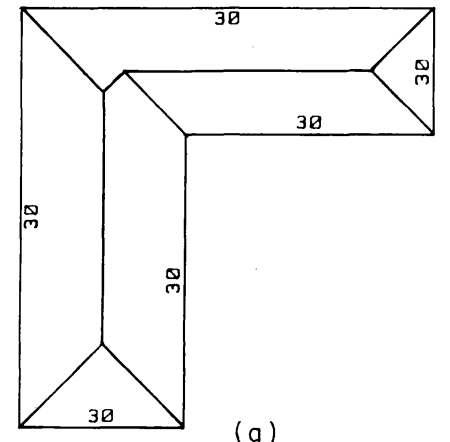

(a)

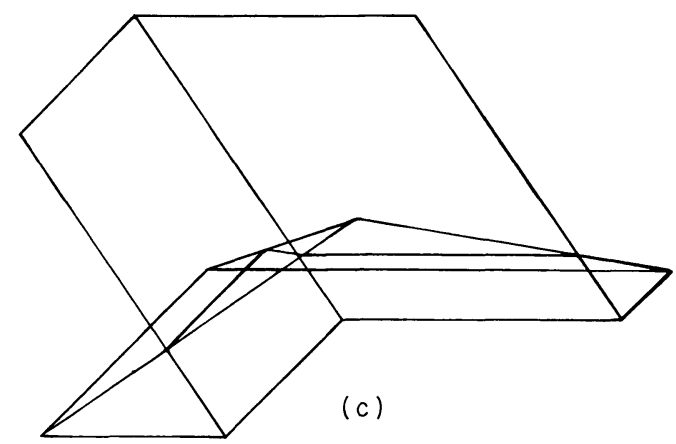

(c) Consejo Superior de Investigaciones Científicas Licencia Creative Commons 3.0 España (by-nc) primer vértice que describe el lado de la base al que corresponde según la secuencia utilizada por el proyectista para describirla. En el caso en que a ese lado de la base le corresponda una pared vertical ( sin faldón de cubierta) deberá darse como inclinación el valor de $90^{\circ}$.

En la Fig. 1 se muestran ejemplos de plantas simple y múltiplemente conexas en las que la numeración en los vértices se hace de acuerdo con el criterio mencionado.

\section{Proceso de cálculo}

El plano que contiene cada faldón queda definido por el punto origen del lado de la base y por el vector perpendicular al plano. La dirección de las limas que delimitan cada faldón puede obtenerse mediante el producto vectorial del vector perpendicular al faldón considerado por los vectores perpendiculares a los faldones contiguos. El procedimiento de cálculo consiste en definir sobre cada faldón un elemento plano auxiliar delimitado por la línea de base y las dos limas. En el caso en el que las limas se corten por encima de la base, este elemento será triangular; en caso contrario, será un cuadrilátero formado por las líneas anteriores y una paralela a la línea de base situada a una altura suficiente, por ejemplo, igual a la máxima dimensión de la planta.

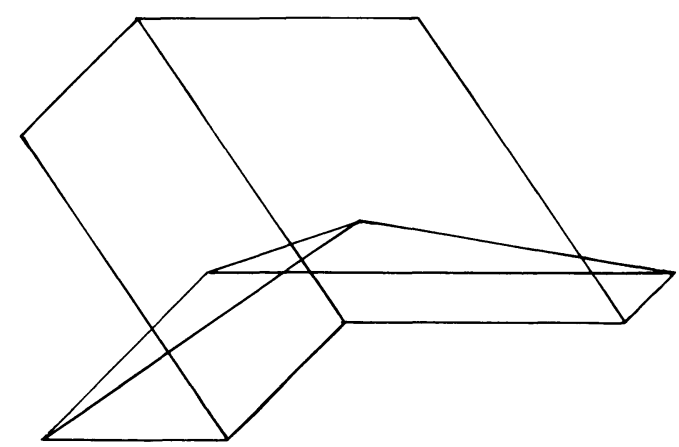

(b)

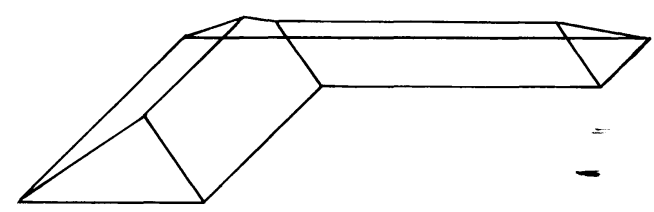

(d) 
La línea de cumbreras correspondiente a cada faldón se determinará hallando las intersecciones de cada uno de estos elementos auxiliares con todos lo demás y reteniendo exclusivamente el polígono más próximo a la base que resulta de considerar el conjunto de todos los cortes.

Considérese el ejemplo de la Fig. 2. Se trata de un edificio con planta en $\mathrm{L}$ como se muestra en $2 \mathrm{a}$, con lados de anchura desigual y que se desea cubrir con faldones igualmente inclinados. En $2 \mathrm{~b}$ puede verse una perspectiva de los elementos auxiliares de todos los faldones, como si estos elementos fuesen de cristal. Los elementos auxiliares correspondientes a los lados de la planta que forman el rincón son trapecios (en este caso paralelogramos), en tanto que los restantes son todos triángulos, de los cuales los correspondientes a los lados más largos sobrepasan las cumbreras, en tanto que los correspondientes a los lados más cortos coinciden con el faldón final. En 2c se han añadido a estos elementos auxiliares las intersecciones entre los mismos en tanto que en $2 \mathrm{~d}$ se presenta una perspectiva del resultado final una vez eliminadas las partes sobrantes de los elementos auxiliares.

En este ejemplo, los cortes entre elementos auxiliares son muy sencillos, pero en la práctica se pueden presentar casos mucho más complicados, tal como el que se va a utilizar para ilustrar la descripción del algoritmo, ejemplo elegido por poder referir al mismo prácticamente todas las situaciones conflictivas que puedan darse en el proceso. Este ejemplo se muestra en la Fig. 3; si destacamos en ella el elemento auxiliar correspondiente a la línea de base regruesada los cortes producidos en el mismo por los restantes elementos se indican en la Fig. 3b. La parte a conservar está representada por la zona rayada de la figura. Por último, en el caso en que existan faldones coplanarios puede ocurrir que los mismos se solapen, tal como ocurre con los faldones correspondientes a los lados regruesados de la planta indicada en la Fig. 4. En tal caso deberán de sustituirse los faldones que presenten solapes por la unión de los mismos, tal como se indica en la Fig. 4b.

Seguidamente, se exponen con sumo detalle los pasos del proceso de cálculo.

A. Determinación de valores geométricos auxiliares. Como se ha indicado cada faldón se asocia con el vértice inicial del correspondiente lado de la base. Para cada uno de los faldones se determinarán los siguientes valores geométricos.

A.1. Cosenos directores del correspondiente lado de la base, para lo cual se dispone de las coordenadas de los vértices inicial y final de dicho lado.

A.2. Componentes del vector perpendicular al faldón. Este vector es perpendicular al correspondiente lado de la base y forma con el eje vertical un ángulo igual al que forma el faldón con el plano horizontal.

A.3. Dirección de la lima correspondiente a cada vértice de la base. Esta dirección se obtiene como producto vectorial de los vectores perpendiculares a los dos faldones que concurren en dicho vértice.

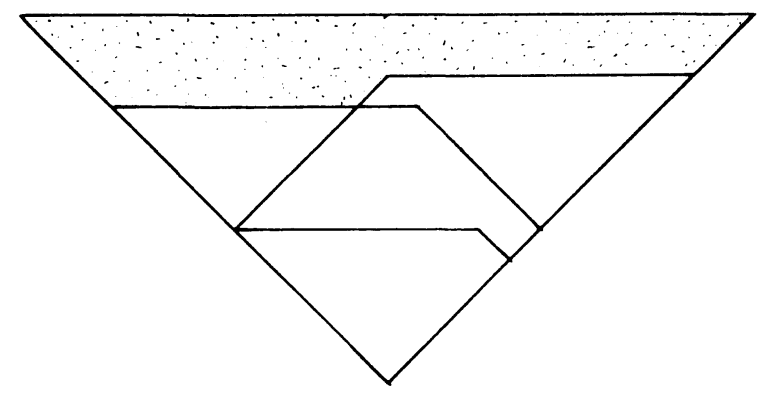

(b) 


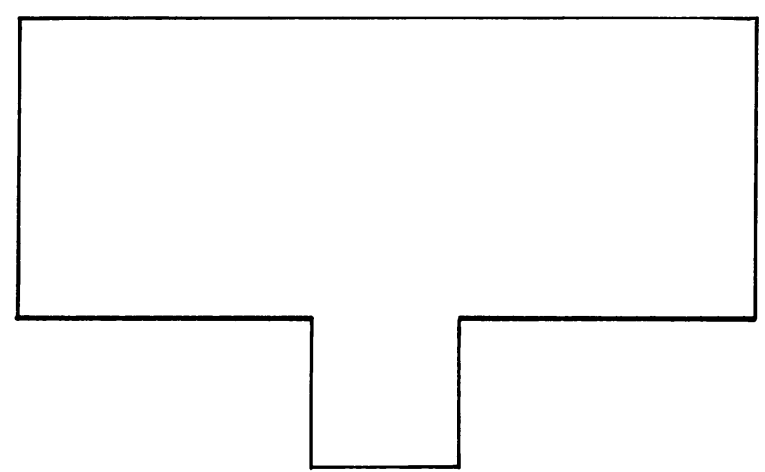

(a)

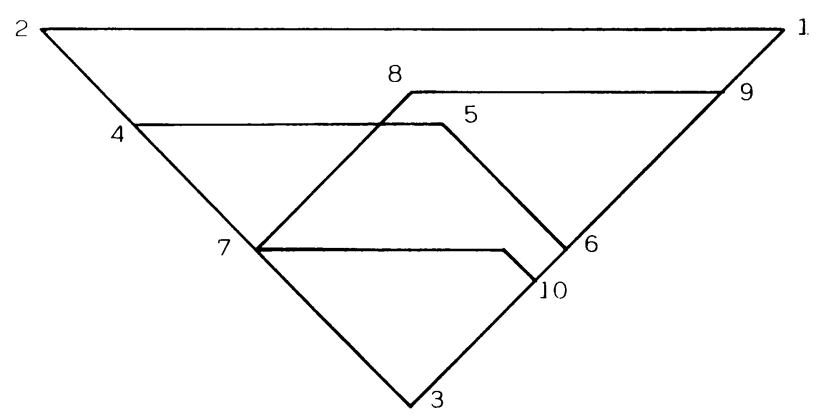

(a)

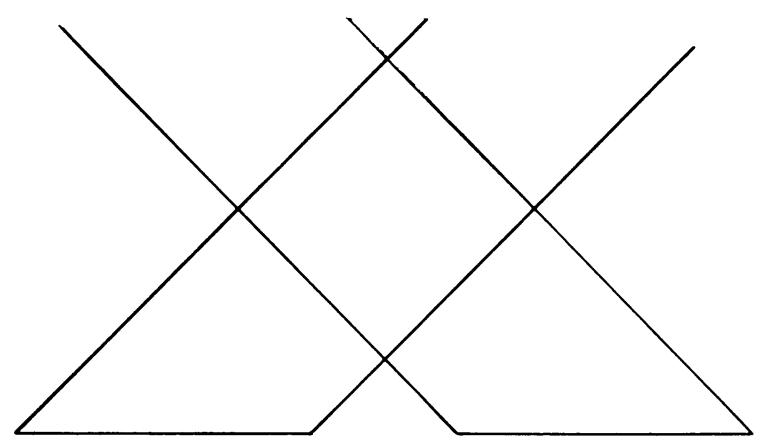

(b)

Fig. 4

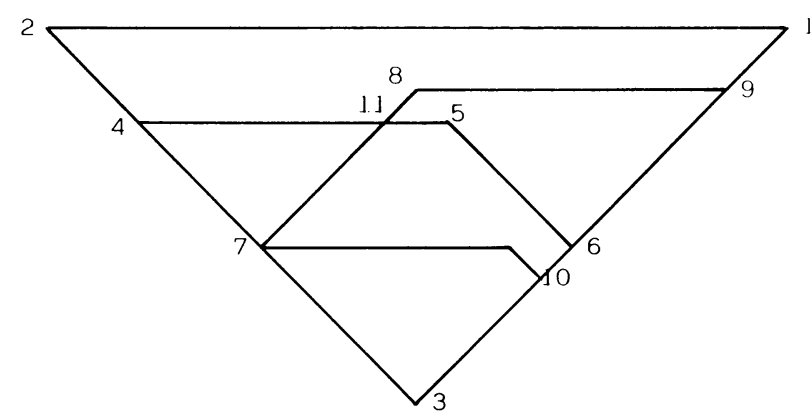

(b)

Fig. 5

B. Definición de los elementos planos auxiliares correspondientes. Para cada faldón se define un elemento auxiliar que contendrá el faldón real en su totalidad. Para definir estos elementos auxiliares se determina en primer lugar la intersección de las limas externas de la arista de base del faldón. Si esta intersección se produce del lado de la arista en que existe el faldón, el elemento auxiliar será el triángulo definido por la arista de base y los trozos de limas comprendidos entre los extremos de la base y el punto de intersección calculada. En el caso en que las limas fuesen paralelas o si su intersección está del lado opuesto al faldón respecto a la arista de base, el elemento auxiliar será un trapecio formado por la arista de la base, los trozos de las limas del lado en que existe el faldón hasta una altura suficientemente grande y una paralela a la base situada a la mencionada cota.

C. Determinación de las intersecciones de los elementos auxiliares. Deben a continuación considerarse las intersecciones de cada uno de estos ele- mentos planos auxiliares con todos los demás. Para cada pareja de elementos que se corten deberá determinarse en primer lugar la intersección de cada elemento con el plano del otro. Seguidamente, retener como intersección real de ambos elementos la parte común de la intersección de cada elemento con el plano del otro. Una vez determinadas todas las intersecciones de un elemento con los restantes, debe comprobarse si estas intersecciones tienen entre sí puntos de contacto o de intersección, debiendo en tal caso separar los cortes en segmentos independientes. Así, si consideramos de nuevo el elemento auxiliar de la Fig. 3d, en la Fig. 5a los cortes producidos sobre este elemento por los restantes son los segmentos 4-5, 5-6, 7-8, 8-9 y 7-10. Como puede verse, los cortes $4-5$ y $7-8$, se intersectan en el punto 11, por lo cual el corte 4-5 debe descomponerse en los cortes 4-11 y 11-5 y el corte 7-8 en los cortes 7-11 y 11-8. De este modo se obtienen ya todos los segmentos que formarán los bordes del faldón solución, esto es, el polígono formado por la secuencia de vértices, 1, 2, 4, 11, 8 y 9. 
D. Reconstrucción del faldón. Esta es la parte más delicada del proceso. Se dispone de la descripción del elemento auxiliar (1, 2, 3 en el ejemplo de la Fig. 5) y de los segmentos de corte (4-11, 11-5, 5-6, 7-11, 11-8, 8-9 y 7-10 en dicho ejemplo). Las etapas de este proceso son como sigue:

D.1. Incorporar como primer elemento de la secuencia el primer vértice del borde inferior (el punto 1 en el ejemplo).

D.2. Recorrer ordenadamente los lados del elemento auxiliar observando si hay cortes con un extremo en dicho lado.

D.2.1. Si no hay ningún corte incorporar a las secuencias solución el punto inicial del lado siguiente (en el primer lado no existe ningún corte, por consiguiente se incorpora a la secuencia el punto 2). Continuar en D.2 con el lado siguiente.

D.2.2. Si hay cortes, elegir el corte más próximo al último vértice de la secuencia solución (en el ejemplo existen los cortes 4-11, 7-10 y 7-11, elegir el corte 4-11). En caso de que haya más de un corte en esta situación, elegir aquel cuyo otro extremo tenga la cota más próxima a la base ( si no existiera el corte 4-11, debería elegirse el corte 7-11 entre los cortes 7-10 y 7-11). Incorporar a la secuencia los extremos de este corte (los puntos 4 y 11 en el ejemplo). Si el último punto incorporado pertenece a otro lado del elemento auxiliar continuar el proceso en el punto D.2.1 con dicho lado (cuando se alcance el punto 9 en el ejemplo se continuará en el punto D.2.1 con el lado 3), en caso contrario continuar en D.2.3.

D.2.3. Buscar todos los cortes que tengan un extremo en el último punto incorporado, elegir para seguir aquel cuyo otro extremo esté próximo a la base (en el ejemplo existen los cortes 11-5, 11-7 y 11-8; deberá elegirse para continuar el 11-8). En el caso en que existan varios cortes que cumplan esta condición, elegir aquel que se aleje menos del origen del último corte en la dirección del mismo ( el ejemplo hubiera sido el simétrico del propuesto tras incorporar el corte 8-11, habría que elegir entre los cortes 11-4 y 11-5, el corte a incorporar debería haber sido 11-4): incorporar el nuevo punto a la secuencia solución. Si dicho punto pertenece a otro lado del elemento, continuar con dicho lado en D.2.1; en caso contrario, continuar en D.2.3 (en el ejemplo se incorporarían los cortes $11-8$ y $8-9$ antes de volver a D.2.1 donde se incorporaría de nuevo el punto 1).
D.3. El faldón queda reconstruido si bien debe prescindirse del último punto, que coincide con el primero (quedará así la secuencia 1, 2, 4, 11, 8 y 9).

E. Unión de faldones coplanarios con solapes. Si al tratar de determinar las intersecciones de cada pareja de elementos auxiliares se detectasen elementos coplanarios, no se determinarán los cortes entre ambos elementos. Sin embargo, una vez concluidas las operaciones del apartado D, se procederá a determinar la posible unión de los faldones resultantes, en caso en que tengan puntos comunes (ver Fig. 5). La determinación de esta unión puede efectuarse utilizando cualquiera de los algoritmos de operaciones gráficas con recintos planos, por ejemplo, (3).

F. Compactación de los resultados. Para compactar los resultados producidos por el algoritmo se deberá, en primer lugar, proceder con cada uno de los faldones a detectar la posible existencia de aristas del mismo en prolongación, eliminando en tal caso los puntos intermedios. Seguidamente, y mediante un procedimiento de ordenación, se identificarán los vértices de los faldones no pertenecientes a la base y cuyas coordenadas coincidan, de modo que se asigne a dichos puntos un mismo número de identificación.

\section{Ejemplos}

Los ejemplos que se incluyen a continuación, muestran algunas de las posibilidades del algoritmo, así como la forma de resolver algunos casos complicados. En cada uno de estos ejemplos se presenta una planta del edifico en la que se reflejan los datos, y una perspectiva de la solución. Sobre cada una de las aristas de los bordes, y del lado del que se extiende la cubierta, figura el ángulo de inclinación del faldón correspondiente; valores positivos de estos ángulos indican que el faldón se eleva sobre la arista en tanto que valores negativos indican que desciende desde la misma. En la perspectiva de la solución se ha dibujado con línea de trazos el contorno de la base, y se presentan todos los contornos de los faldones de la cubierta como si fuera un modelo de alambres.

El ejemplo de la Fig. 6 corresponde a uno de los casos más simples, una cubierta a cuatro aguas sobre una planta rectangular teniendo uno de los faldones mayor inclinación qué los restantes, lo que produce un resultado asimétrico. El ejemplo de la Fig. 7 corresponde a una cubierta a dos aguas sobre una planta cuadrada pero con la 

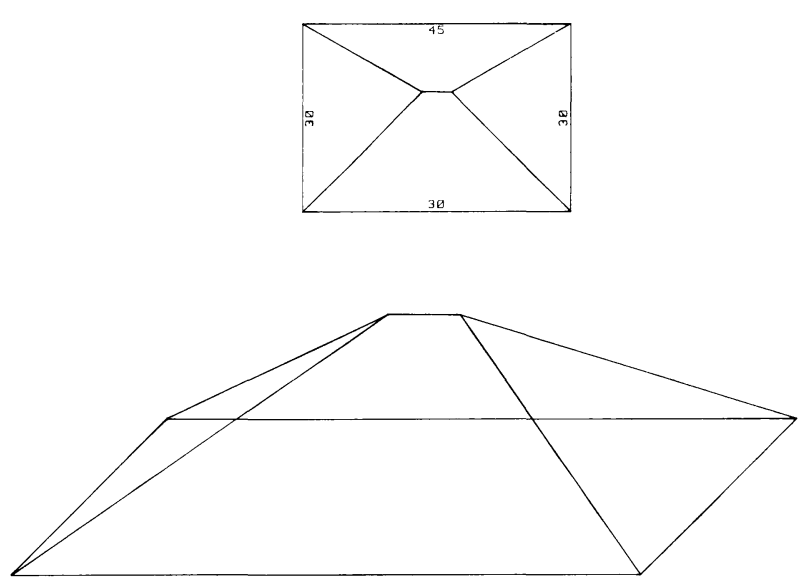

Iig. ()
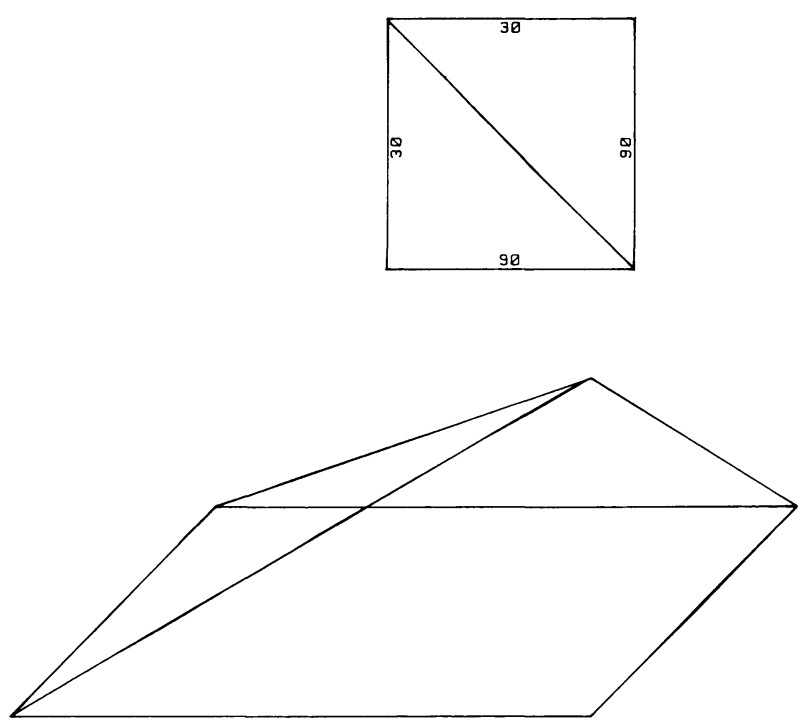

Fig. 7
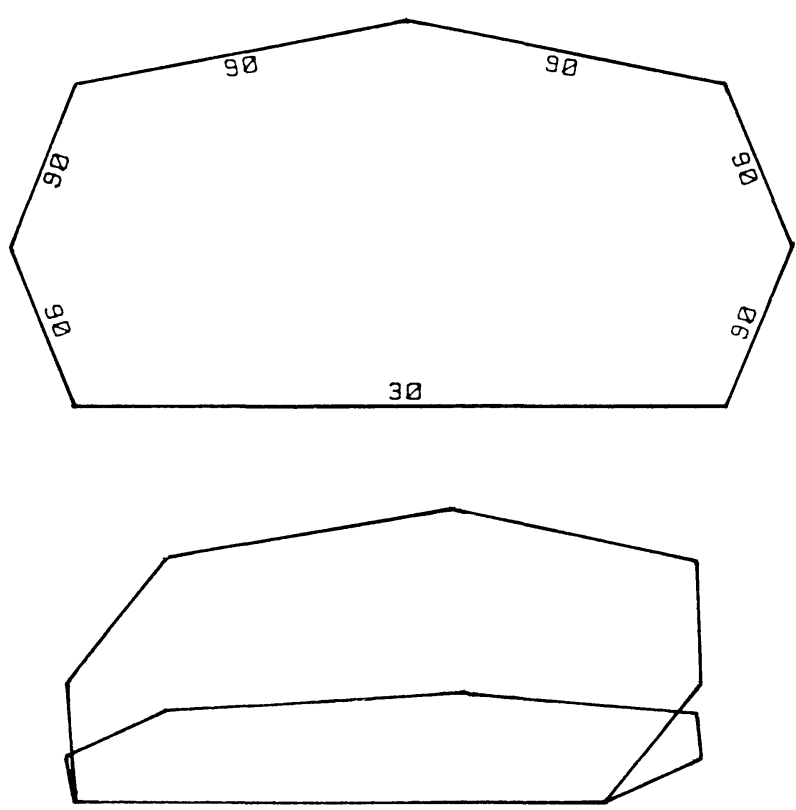

Fig. S

(c) Consejo Superior de Investigaciones Científicas Licencia Creative Commons 3.0 España (by-nc)
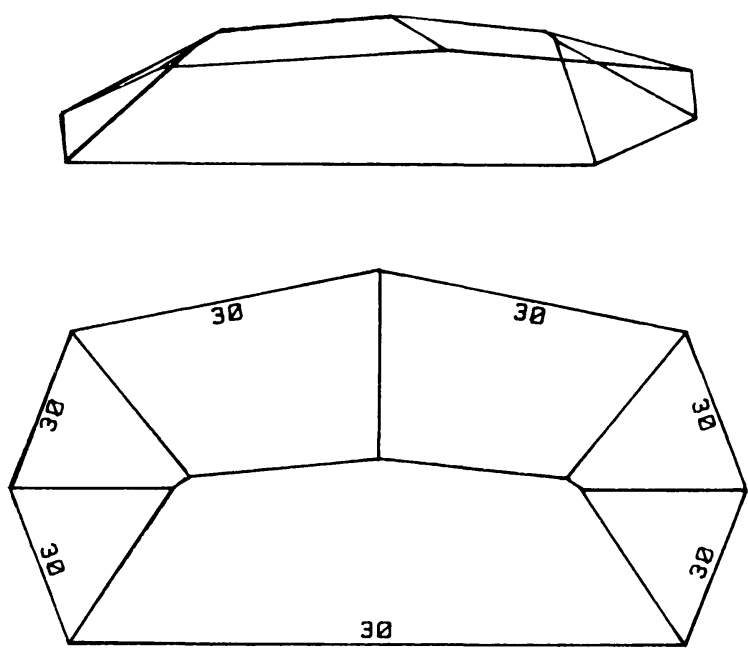

lis. ()
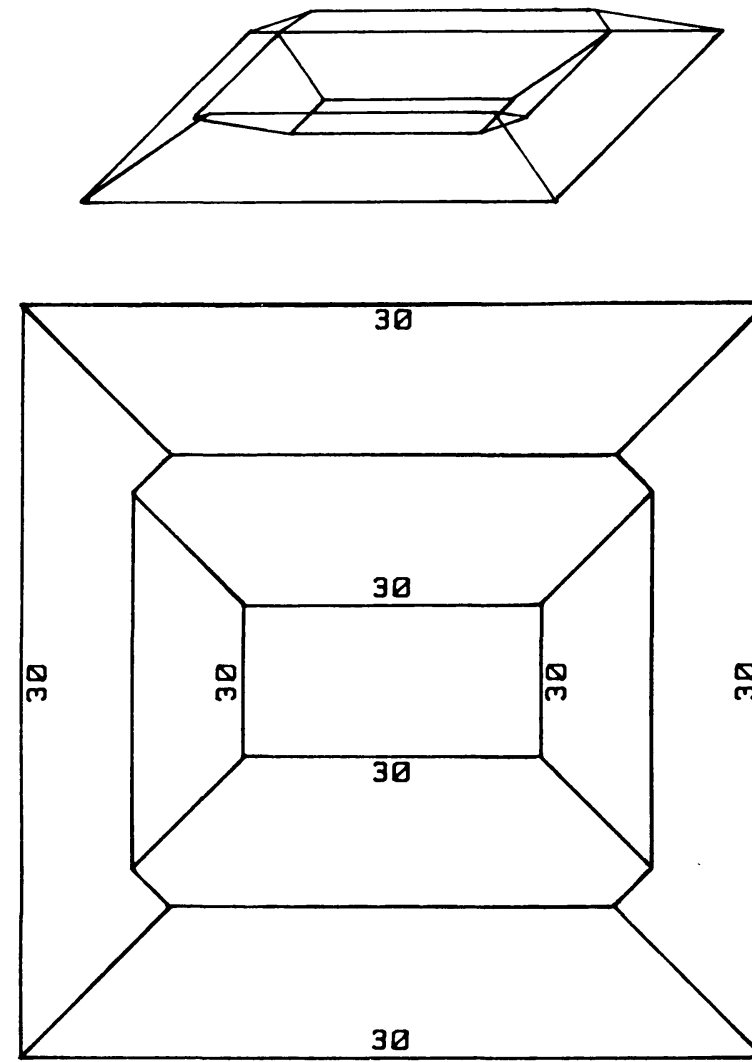

Fig. 10

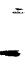

http://informesdelaconstruccion.revistas.csic.es 

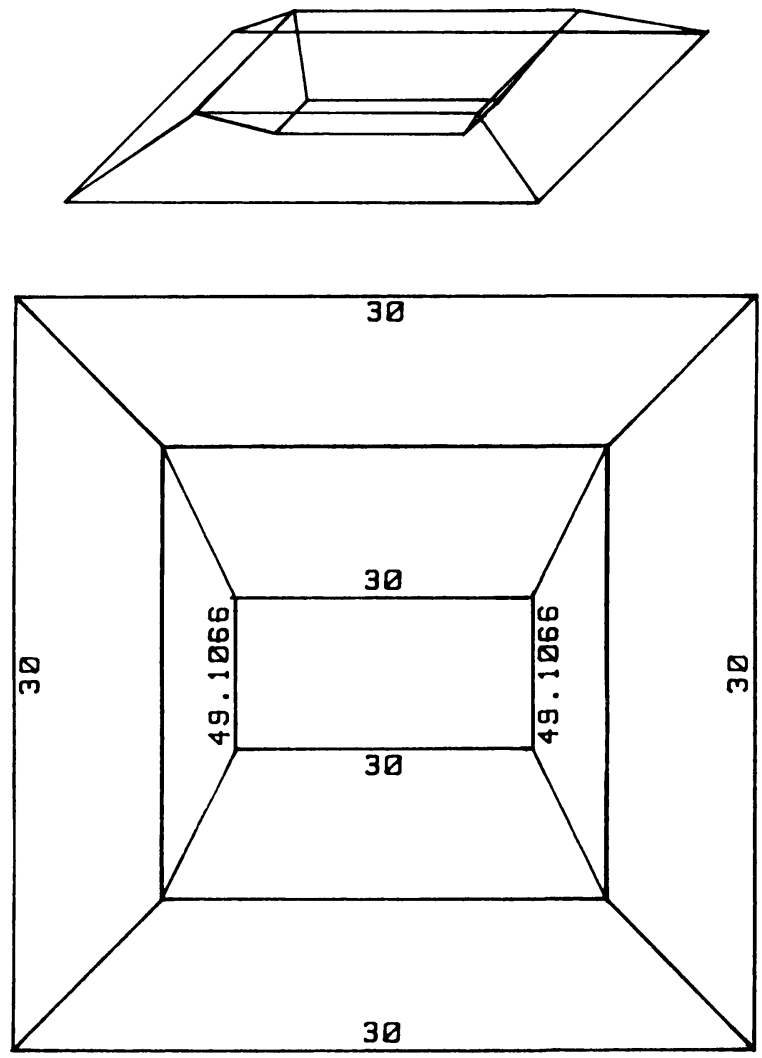

Fig. 11
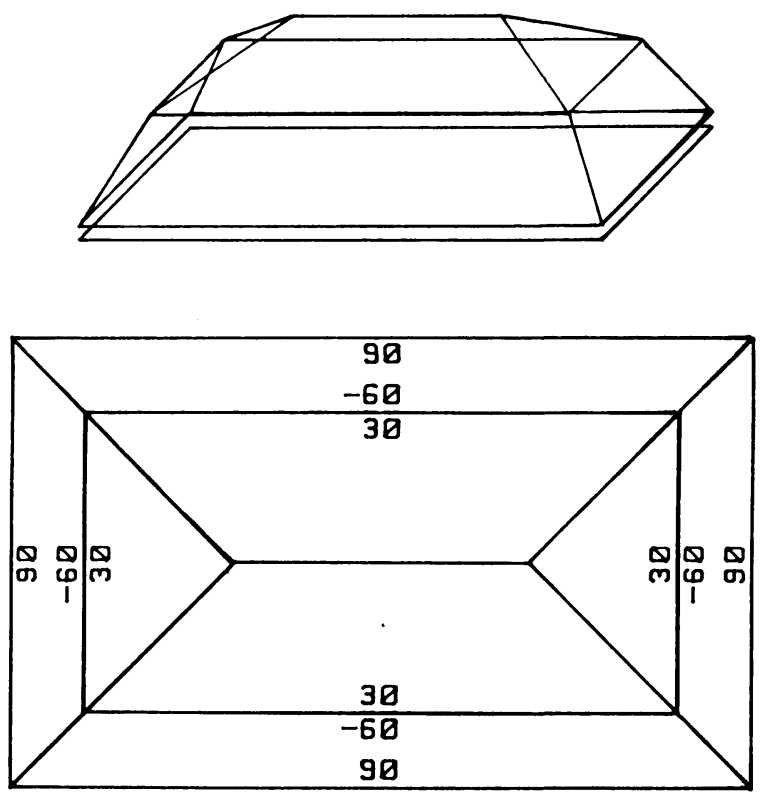

Fig. 12
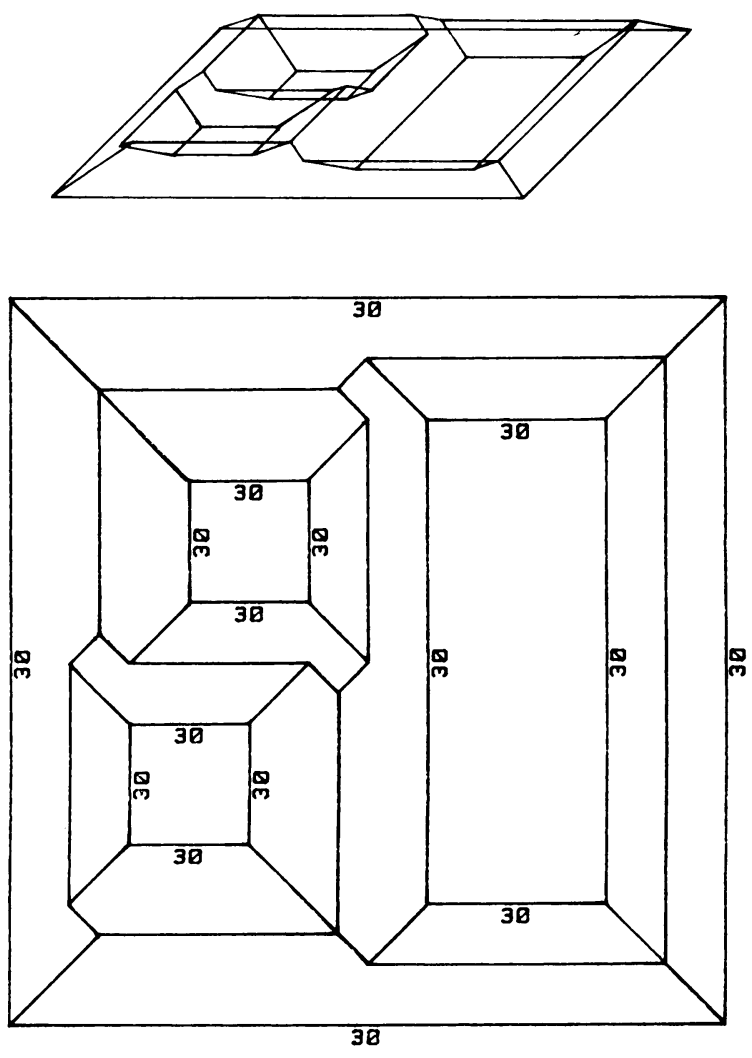

I.1. 1.;
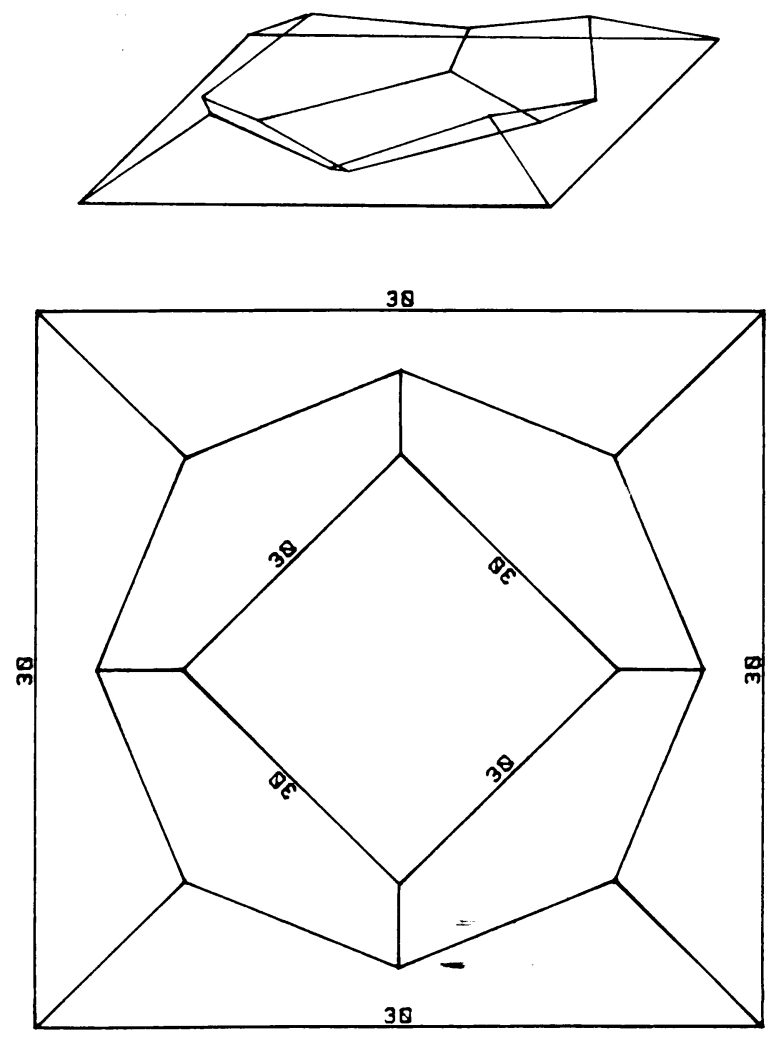

lig. 11

(c) Consejo Superior de Investigaciones Científicas Licencia Creative Commons 3.0 España (by-nc)

http://informesdelaconstruccion.revistas.csic.es 
cumbrera en diagonal e inclinada. El ejemplo de la Fig. 8 corresponde a una cubierta inclinada de un solo faldón sobre una planta simple; se trata de un caso que pese a su aparente sencillez no es resuelto correctamente por alguno de los programas existentes. El ejemplo de la Fig. 9 corresponde a una cubierta con faldones igualmente inclinados sobre una planta poligonal irregular, produciéndose en este caso cumbreras inclinadas. Los ejemplos de las Figs. 10 y 11 corresponden ambos a un edificio con un patio interior y lados de ancho desigual. En la Fig. 10, con faldones igualmente inclinados se presentan cumbreras con distinta cota, en tanto que en la Fig. 11 se presenta una solución con cumbreras al mismo nivel y con los faldones exteriores con la misma inclinación, teniendo en consecuencia los faldones que dan al patio inclinaciones diferentes. El ejemplo de la Fig. 12 corresponde a una cubierta amansardada; este ejemplo podría haberse resuelto con otro conjunto de datos, pero se ha elegido la presente forma para utilizar inclinaciones negativas de los faldones. El ejemplo de la Fig. 13 muestra la cubierta de un edificio con tres patios interiores. Por último, en la Fig. 14 se muestra la cubierta para un edificio de planta cuadrada con un patio, también de planta cuadrada, pero girada $45^{\circ}$, con lo que se produce una cubierta en forma de corona.

\section{Conclusiones}

El algoritmo que se describe, permite resolver la mayor parte de los casos prácticos de cubiertas inclinadas, que se presentan en el proyecto de edificios. Los datos necesarios son los mínimos imprescindibles y la solución producida es compacta y simple de interpretar, lo cual permite la utilización del algoritmo por cualquier programa de diseño asistido de edificios.

Se ha señalado que este algoritmo permite resolver la mayor parte de los casos prácticos. Entre los casos que no permite tratar puede citarse aquel en el que algún faldón no fuese simplemente conexo o aquel en el que el primer vértice del lado de la base de algún faldón no perteneciese al faldón definitivo. La consideración de estos casos no presenta mayores complicaciones teóricas, pero su consideración complicaría innecesariamente tanto los datos a suministrar al algoritmo como la forma de presentar los resultados. Estos casos si bien pueden presentarse en teoría, son difícilmente imaginables como casos prácticos en arquitectura.

\section{REFERENCIAS}

(1) Recuero, A., y Gutiérrez, J.P.: Perspective Drawing of Three-Dimensional Objects, Microsofware for Engineers, 1987, vol. 3, n. ${ }^{\circ}$, pp. 79-84.

(2) Recuero, A.; Gutiérrez, J.P., y O. Río: Dibujo de perspectivas, asistido por computador, utilizando dispositivos trazadores gráficos. Monografía del Instituto Eduardo Torroja. n. ${ }^{\circ} 391$, diciembre 1987.

(3) Recuero, A., y Gutiérrez, J.P.: Computerized Logical Operations with Polygonal Domains. Microcomputer in Engineering. Pineridge Press. Swansea 1986.

(4) Recuero, A., y Gutiérrez, J.P.: Automatic Generation of Sloped Roofs over Polygonal Plants, Proceedings. Compugraphics'91. Ed. Harold P. Santo, 1991, vol. 2, pp. 334-343. 